\title{
Investigation of inhibitory effects on EPC-mediated neovascularization by different bisphosphonates for cancer therapy
}

\author{
THOMAS ZIEBART ${ }^{1}$, JOHANNA ZIEBART ${ }^{1,3}$, LEONIE GAUSS $^{1}$, ANDREAS PABST $^{1}$, \\ MAXIMILIAN ACKERMANN ${ }^{2}$, RALF SMEETS ${ }^{4}$, MORITZ A. KONERDING $^{2}$ and CHRISTIAN WALTER ${ }^{1}$ \\ ${ }^{1}$ Department of Maxillofacial Surgery, ${ }^{2}$ Institute of Functional and Clinical Anatomy and ${ }^{3}$ Department of Prosthodontics, \\ University Medical Center of Johannes Gutenberg University Mainz, D-55131 Mainz; ${ }^{4}$ Department of Maxillofacial Surgery, \\ University Medical Center of Hamburg-Eppendorf, Hamburg, Germany
}

Received May 25, 2012; Accepted July 11, 2012

DOI: 10.3892/br.2013.145

\begin{abstract}
Bisphosphonates (BPs) are potent drugs, used in metastatic cancer-like prostate or breast carcinoma. In recent studies, besides reduced bone remodeling, influences on angiogenesis and neovascularization were reported. Since BPs have the tendency to accumulate in the bones, the biological effect of various nitrogen- and non-nitrogen BPs on endothelial progenitor cells (EPCs) that originated from bone marrow and mobilized under physiological and pathophysiological conditions, such as tumor neovascularization, was investigated. EPCs subsequent to 72-h treatment with different concentrations of bisphosphonates comprised the non-nitrogen-containing BP clodronate and the nitrogen-containing BPs ibandronate, pamidronate and zoledronate. After incubation, biological activity was measured by using the migration boyden chamber assay and measurement of the colony-forming ability. Nitrogen-containing BPs inhibited the migration ability and differentiation of EPCs in a dose-dependent manner, as compared to the non-treated control groups. More specifically, the nitrogen-containing BP zoledronate significantly inhibited angiogenesis and neovascularization. Clodronate was less distinct on EPC function. To underline the importance of neovascularization in the context of tumor angiogenesis, EPC functions were significantly influenced in a dose-dependent manner by nitrogen-containing BPs. From these findings, we conclude that especially the nitrogencontaining BPs, such as zoledronate, are potential anticancer agents through the inhibition of neovascularization.
\end{abstract}

Correspondence to: Dr Thomas Ziebart, Department of Maxillofacial Surgery, University Medical Center of Johannes Gutenberg University Mainz, Augustusplatz 2, D-55131 Mainz, Germany E-mail: ziebart@mkg.klinik.uni-mainz.de

Key words: bisphosphonate, endothelial progenitor cells, endothelial progenitor cell, neovascularization, angiogenesis, cancer

\section{Introduction}

The benefit of bisphosphonates (BPs) is unequivocal in the treatment of malignant bone neoplasias, such as multiple myeloma, bone metastases (e.g., due to primary breast or prostate cancer) or metabolic bone diseases, such as Paget's disease and severe osteoporosis.(1). Their anti-resorptive mechanism results in a significant reduction of skeletal-related events such as pain, hypercalcaemic episodes or fractures that require radiation therapy or stabilizing operations, thus improving the quality of life (2). BPs mainly inhibit bone resorption through selective adsorption on mineral surfaces and subsequent internalization via the bone-resorptive activity of osteoclasts which interfere with various biochemical processes. The simpler, non-nitrogen-containing BPs, such as clodronate, have the potential to be metabolically incorporated into nonhydrolysable analogues of adenosine triphosphate (ATP) that inhibit ATP-dependent intracellular enzymes. By contrast, the more potent, nitrogen-containing BPs, such as ibandronate, pamidronate and zoledronate, inhibit farnesyl pyrophosphate synthase, a key enzyme of the mevalonate pathway, thereby preventing the biosynthesis of isoprenoid compounds that are essential for the post-translational modification of small guanosine triphosphate (GTP)-binding proteins (that are also GTPases), such as Rab, Rho and Rac (3-5). The inhibition of protein prenylation and the disruption of the function of these key regulatory proteins explain the loss of osteoclast activity (6).

Besides the inhibition of osteoclast function, an antiangiogenic effect of BPs has been throroughly investigated (7-9). Sufficient tumor angiogenesis and vessel formation is indispensable for tumor growth and progression $(10,11)$. In this context, the term angiogenesis refers to sprouting from pre-existing vessels of rather mature endothelial cells. Apart from this bone-marrow-derived mechanism, circulating endothelial progenitor cells (EPCs) bear the potential of the de novo creation of primordial vessels, termed vasculogenesis (12-14). Besides this biological function, EPCs demonstrated strong paracrine effects in terms of the production of several cytokines and growth factors, such as vascular endothelial 


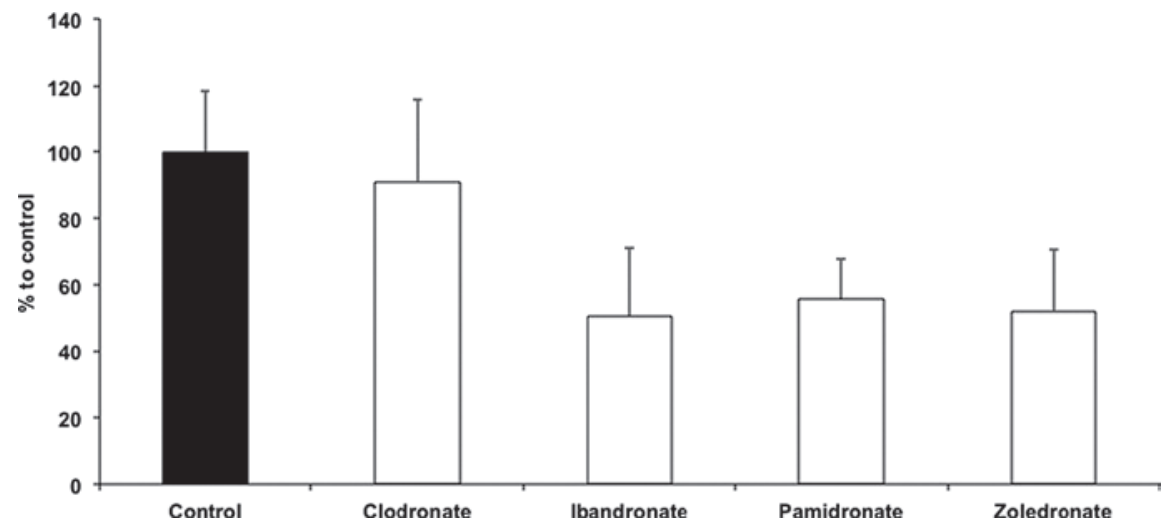

Figure 1. Migration of EPCs affected by nitrogen- and non-nitrogen-containing bisphosphonates. Significantly reduced migration ability of EPCs subsequent to treatment with nitrogen-containing BPs compared with the non-BP-treated control group and EPCs treated with clodronate.

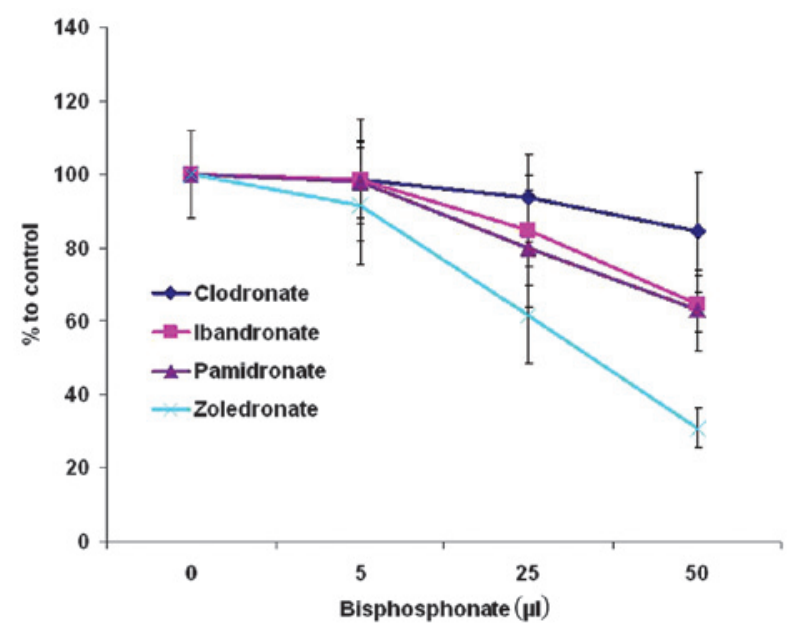

Figure 2. Number of CFU under treatment of different bisphosphonates. Dose reduced number of CFU after treatment with nitrogen-containing BPs. Strongest negative effect was observed after zoledronate treatment.

growth factor (VEGF), stromal cell-derived factor-1, insulinlike growth factor-1 and hepatocyte growth factor that increase angiogenesis and neovascularization (15). EPCs have been demonstrated to support short-, as well as long-term neovascularization (14). The aim of this study was to investigate the possible inhibitory effect of different BPs on EPC-mediated neovascularization as a possible new target for cancer therapy.

\section{Materials and methods}

EPC culture assay. Mononuclear cells (MNCs) were isolated by density gradient centrifugation with Biocoll (Biochrom KG, Berlin, Germany) from the peripheral blood of healthy human volunteers as previously described (14). Immediately following isolation, total MNCs ( $8 \times 10^{6}$ cells $/ \mathrm{ml}$ medium) were plated on $25-\mathrm{cm}^{2}$ culture flasks coated with human fibronectin (Sigma, Steinheim, Germany) and maintained in endothelial basal medium (EBM) supplemented with EGM SingleQuots, VEGF (100 ng/ml), and 20\% FCS for 3 days. EPCs were obtained as a byproduct from blood donors at our University hospital therefore no approval from the ethics committee was required. This was an in vitro study and only cell culture experiments were conducted.
Migration assay. To examine the effect of bisphosphonates on EPC migration, a 24-well Boyden chamber assay system (ThinCert ${ }^{\mathrm{TM}}$ ) was used, according to the manufacturer's instructions (Greiner Bio-One, Frickenhausen, Germany). EPCs were incubated for $24 \mathrm{~h}$ with varying concentrations of bisphosphonates. Cells were harvested, washed twice in PBS and resuspended in EPC medium for adjustment to a final concentration of $10^{6} \mathrm{ml}^{-1}$. EPCs were stimulated to migrate from the upper to the lower chambers subsequent to adding $10 \mathrm{ng} / \mathrm{ml} \mathrm{VEGF}$ to the lower chambers. After $12 \mathrm{~h}$, the cells were stained with fluorescent dye calcein-AM. The culture medium was then removed from the inserts, while the inserts were transferred onto a freshly prepared 24-well plate, containing $500 \mu \mathrm{l}$ trypsin-EDTA/well. This plate was incubated for $10 \mathrm{~min}$ in a cell culture incubator at $37^{\circ} \mathrm{C}$ with $5 \% \mathrm{CO}_{2}$ with sporadic agitation. The inserts were discarded and $200 \mu \mathrm{l}$ of the trypsin-EDTA solution, now containing the detached migratory cells, was transferred to a flat-bottom, black 24-well plate. A fluorescence plate reader was used for quantification.

Colony-forming unit assay. To examine the effect of the colony-forming ability, EPCs were cultured in MethoCult ${ }^{\mathrm{TM}}$ 
GF H84434 culture medium (STEMCELL Technologies, Inc., Vancouver, BC, Canada) on 35-mm well culture plates for 14 days, according to the manufacturer's instructions. Cultures were incubated at $37^{\circ} \mathrm{C}$ with $5 \% \mathrm{CO}_{2}$, and scored for colony formation after 14 days.

Statistical analysis. Continuous variables were presented as the mean \pm SEM. Comparisons between groups were analyzed by analysis of variance (ANOVA, post hoc test, Tukey) for experiments with $>2$ subgroups, or the Student's t-test (twosided). The software SPSS 16.0 for Windows was used for calculations. $\mathrm{P}<0.05$ was considered to indicate statistically significant differences.

\section{Results}

Migration. Results of the control group were set to $100 \%$. EPC migration was not reduced subsequent to clodronate treatment (96.32 $\pm 14.61 \%)$ compared to the control group. However, treatment with ibandronate $(45.5 \pm 6.53 \%)$, pamidronate $(53.95 \pm 6.24 \%)$ and zoledronate $(51.29 \pm 5.07 \%)$ significantly affected the migration of EPCs $(\mathrm{P}<0.01)$. No differences were observed in the migration ability in the non-nitrogen BP clodronate group (Fig. 1).

Colony-forming units. Fig. 2 shows the colony-forming unit (CFU) ability during BP treatment. At a concentration of $5 \mu \mathrm{mol}$ BPs, no significant differences were observed between the control group $(57.59 \pm 0.55)$, clodronate $(55.22 \pm 0.44)$, ibandronate $(55.11 \pm 3.97)$, pamidronate $(54.88 \pm 3.27)$ and zoledronate (51.22 \pm 1.92$)$. Moreover, $25 \mu \mathrm{M}$ clodronate $(53.22 \pm 4.2)$ did not demonstrate statistically significant differences when compared to the control group. A tendency for reduced CFU ability was observed at $25 \mu \mathrm{M}$ ibandronate $(48.22 \pm 5.71)$ and pamidronate $(45.44 \pm 4.77)$. Zoledronate $(35.11 \pm 3.73)$ showed statistically significant $\mathrm{CFU}$ results. Increased BP concentrations of $50 \mu \mathrm{M}$ showed notable inhibitory effects on CFU regarding nitrogen-containing BPs ibandronate (38.66 \pm 1.52$)$, pamidronate $(37.55 \pm 2.66)$ and zoledronate $(18.55 \pm 2.11)$. No significant difference was found between ibandronate and pamidronate. The most marked negative effect on CFU was found for zoledronate, while the lowest was for clodronate.

\section{Discussion}

BPs are common anti-bone-resorptive drugs with clinical effectiveness against various types of cancer, when used in combination with other conventional anticancer drugs (16-18). Apart from their direct effect on cancer cells, these drugs are believed to affect angiogenesis (19). Tumors and ischemic tissue have the potential to recruit EPCs from the bone marrow in order to increase the number of sprouting vessels for blood supply by indirect effects, such as paracrine secretion of growth factors including VEGF or the direct differentiation of EPCs in mature endothelial cells $(20,21)$. In patients with head and neck cancer, for example, an elevated EPC concentration in peripheral venous blood was demonstrated (22).

Therefore, the effect of different BPs on EPCs as an option for cancer therapy was investigated. Consistent with previous studies, results showed that the EPC concentration is reduced by different BPs in a dose-dependent manner $(8,9)$. Particularly, pamidronate and zoledronate had a significant impact on cell numbers compared to the control and clodronate groups. Yamada et al (8) showed increased EPC apoptosis rate subsequent to zoledronate treatment in vitro. In this study, important biological functions, such as migration and stem cell differentiation were demonstrated to be impaired by BPs. Reduced migration capacity resulted in impaired mobilization from bone marrow niche and homing of EPC $(23,24)$. EPC differentiation is a pre-condition for clonal expansion and stem cell function. The nitrogen-containing BP zoledronate was found to have a significant negative effect on colony formation, while no effect was detected by the non-nitrogen containing BP clodronate. Nitrogen-containing and non-nitrogen-containing BPs have different ways of action resulting in varying effects of BPs. Non-nitrogen-containing BPs, such as clodronate, are built into non-hydrolysable ATP that inhibit many different ATP-dependent intracellular enzymes (25). Therefore, extremely high concentrations are needed to completely inhibit special pathways. By contrast, the nitrogen-containing BPs, such as pamidronate and zoledronate, inhibit farnesyl pyrophosphate synthase, a key enzyme of the mevalonate pathway (26). This pathway is significant for the production of small $\mathrm{G}$ proteins, such as Ras, Rac and Rho proteins, which are important for intracellular structure and mechanisms, such as intracellular transport $(27,28)$, and may contribute to the greater impact of nitrogen-containing BPs.

In conclusion, within the boundaries of in vitro studies, these data support the theory of the anti-angiogenetic component of nitrogen-containing BPs. Investigations regarding the effect of BPs on the biology of EPC are likely to provide insights into the role of BPs in inhibiting tumor vasculogenesis, as well as a possible therapeutic option in the future.

\section{References}

1. Neville-Webbe HL and Coleman RE: Bisphosphonates and RANK ligand inhibitors for the treatment and prevention of metastatic bone disease. Eur J Cancer 46: 1211-1222, 2010.

2. Coleman RE: Metastatic bone disease: clinical features, pathophysiology and treatment strategies. Cancer Treat Rev 27: 165-176, 2001.

3. Rogers MJ: New insights into the molecular mechanisms of action of bisphosphonates. Curr Pharm Des 9: 2643-2658, 2003.

4. Rogers MJ, Gordon S, Benford HL, et al: Cellular and molecular mechanisms of action of bisphosphonates. Cancer 88: 2961-2978, 2000.

5. Dunford JE, Thompson K, Coxon FP, et al: Structure-activity relationships for inhibition of farnesyl diphosphate synthase in vitro and inhibition of bone resorption in vivo by nitrogen-containing bisphosphonates. J Pharmacol Exp Ther 296: 235-242, 2001.

6. Fisher JE, Rogers MJ, Halasy JM, et al: Alendronate mechanism of action: geranylgeraniol, an intermediate in the mevalonate pathway, prevents inhibition of osteoclast formation, bone resorption, and kinase activation in vitro. Proc Natl Acad Sci USA 96: 133-138, 1999.

7. Santini D, Vincenzi B, Avvisati G, et al: Pamidronate induces modifications of circulating angiogenetic factors in cancer patients. Clin Cancer Res 8: 1080-1084, 2002.

8. Yamada J, Tsuno NH, Kitayama J, et al: Anti-angiogenic property of zoledronic acid by inhibition of endothelial progenitor cell differentiation. J Surg Res 151: 115-120, 2009.

9. Ziebart T, Pabst A, Klein MO, et al: Bisphosphonates: restrictions for vasculogenesis and angiogenesis: inhibition of cell function of endothelial progenitor cells and mature endothelial cells in vitro. Clin Oral Investig 15: 105-111, 2011.

10. Kerbel RS: Tumor angiogenesis. N Engl J Med 358: 2039-2049, 2008. 
11. Rak JW, St Croix BD and Kerbel RS: Consequences of angiogenesis for tumor progression, metastasis and cancer therapy. Anticancer Drugs 6: 3-18, 1995.

12. Asahara T, Masuda $\mathrm{H}$, Takahashi $\mathrm{T}$, et al: Bone marrow origin of endothelial progenitor cells responsible for postnatal vasculogenesis in physiological and pathological neovascularization. Circ Res 85: 221-228, 1999.

13. Asahara T, Murohara T, Sullivan A, et al: Isolation of putative progenitor endothelial cells for angiogenesis. Science 275 964-967, 1997.

14. Ziebart T, Yoon CH, Trepels T, et al: Sustained persistence of transplanted proangiogenic cells contributes to neovascularization and cardiac function after ischemia. Circ Res 103: 1327-1334, 2008.

15. Urbich C, Heeschen C, Aicher A, et al: Cathepsin L is required for endothelial progenitor cell-induced neovascularization. Nat Med 11: 206-213, 2005.

16. Efstathiou E, Bozas G, Kostakopoulos A, et al: Combination of docetaxel, estramustine phosphate, and zoledronic acid in androgen-independent metastatic prostate cancer: efficacy, safety, and clinical benefit assessment. Urology 65: 126-130, 2005.

17. Bertelli G, Heouaine A, Arena G, et al: Weekly docetaxel and zoledronic acid every 4 weeks in hormone-refractory prostate cancer patients. Cancer Chemother Pharmacol 57: 46-51, 2006.

18. Vogt U, Bielawski KP, Bosse U and Schlotter CM: Breast tumour growth inhibition in vitro through the combination of cyclophosphamide/metotrexate/5-fluorouracil, epirubicin/cyclophosphamide, epirubicin/paclitaxel, and epirubicin/docetaxel with the bisphosphonates ibandronate and zoledronic acid. Oncol Rep 12: 1109-1114, 2004.

19. Wood J, Bonjean K, Ruetz S, et al: Novel antiangiogenic effects of the bisphosphonate compound zoledronic acid. J Pharmacol Exp Ther 302: 1055-1061, 2002.
20. Janic B and Arbab AS: The role and therapeutic potential of endothelial progenitor cells in tumor neovascularization. Sci World J 10: 1088-1099, 2010.

21. De Falco E, Porcelli D, Torella AR, et al: SDF-1 involvement in endothelial phenotype and ischemia-induced recruitment of bone marrow progenitor cells. Blood 104: 3472-3482, 2004.

22. Brunner M, Thurnher D, Heiduschka G, Grasl M, Brostjan C and Erovic BM: Elevated levels of circulating endothelial progenitor cells in head and neck cancer patients. J Surg Oncol 98: 545-550, 2008.

23. Aicher A, Zeiher AM and Dimmeler S: Mobilizing endothelial progenitor cells. Hypertension 45: 321-325, 2005.

24. Hristov M, Erl W and Weber PC: Endothelial progenitor cells: mobilization, differentiation, and homing. Arterioscler Thromb Vasc Biol 23: 1185-1189, 2003.

25. Frith JC, Monkkonen J, Blackburn GM, Russell RG and Rogers MJ: Clodronate and liposome-encapsulated clodronate are metabolized to a toxic ATP analog, adenosine 5'-(beta, gamma-dichloromethylene) triphosphate, by mammalian cells in vitro. J Bone Miner Res 12: 1358-1367, 1997.

26. Nogawa M, Yuasa T, Kimura S, et al: Zoledronic acid mediates Ras-independent growth inhibition of prostate cancer cells. Oncol Res 15: 1-9, 2005

27. Bifulco M: Role of the isoprenoid pathway in ras transforming activity, cytoskeleton organization, cell proliferation and apoptosis. Life Sci 77: 1740-1749, 2005.

28. Crick DC, Andres DA and Waechter CJ: Novel salvage pathway utilizing farnesol and geranylgeraniol for protein isoprenylation. Biochem Biophys Res Commun 237: 483-487, 1997. 